\title{
La enfermedad renal crónica en el Perú. Una revisión narrativa de los artículos científicos publicados
}

\section{Chronic kidney disease in Peru. A narrative review of scientific papers published}

Correspondencia Percy Herrera-Añazco silamud@gmail.com

Recibido: 23/05/2016 Arbitrado por pares Aprobado: 15/06/2016

Citar como: Herrera-Añazco $P$, Pacheco-Mendoza J, Taype-Rondan A. La enfermedad renal crónica en el Perú. Una revisión narrativa de los artículos científicos publicados. Acta Med Peru. 2016;33(2):130-7
Percy Herrera- Añazco ${ }^{1,2}$, Josmel Pacheco-Mendoza ${ }^{3, a}$, Alvaro TaypeRondan ${ }^{4}$

1 Servicio de Nefrología, Hospital Nacional Dos de Mayo. Lima, Perú

2 Escuela de Medicina, Universidad Peruana de Ciencias Aplicadas. Lima, Perú

3 Universidad San Ignacio de Loyola. Lima, Perú.

4 CRONICAS Center of Excellence in Chronic Diseases, Universidad Peruana Cayetano Heredia. Lima, Perú.

a Médico Veterinario

\section{RESUMEN}

Se describe la producción científica peruana que sobre enfermedad renal crónica (ERC) se ha escrito en la literatura médica mediante una revisión bibliográfica no sistemática hasta abril del 2016. Los resultados se dividieron en seis áreas temáticas: prevalencia de ERC, etiología de la ERC, diagnóstico y manejo, cobertura de la atención, costos de la atención y mortalidad. Se encontraron 39 artículos, pero solo 16 se utilizaron para el análisis. La prevalencia de la ERC en algunas regiones del país llega a 16\%. La diabetes y la glomerulonefritis son las causas más frecuentes ERC en hemodiálisis (HD). En Lima y Callao, menos del $9 \%$ de pacientes diabéticos tienen albuminuria en su evaluación. El despistaje de ERC en pacientes hipertensos es costo efectivo. Menos del $50 \%$ de la población que requiere HD la está recibiendo. La mortalidad de población incidente en HD puede llegar hasta el $50 \%$ a los siete meses. La principal causa de muerte es cardiovascular. En conclusión, La ERC es una patología frecuente, con problemas en el diagnóstico y tratamiento temprano. Hay problemas de cobertura de HD y la mortalidad en HD es alta.

Palabras clave:

Insuficiencia renal crónica; Epidemiología; Diálisis renal (fuente: DeCS BIREME). 


\begin{abstract}
We describe the Peruvian scientific production on chronic kidney disease (CKD) that has been written in the medical literature by performing a non-systematic literature review up to April 2016. The results were divided into six thematic areas: prevalence of CKD, etiology of CKD, diagnosis and management, care coverage, care costs and mortality. Thirtynine papers were found, but only 16 were used for analysis. The prevalence of CKD in some Peruvian regions reaches $16 \%$. Diabetes and glomerulonephritis are the most common causes for CKD requiring hemodialysis (HD). In Lima and Callao, less than $9 \%$ of diabetic patients have albuminuria in their evaluation. Screening for CKD in hypertensive patients is cost-effective. Less than $50 \%$ of the population requiring $\mathrm{HD}$ is actually undergoing this procedure. The mortality rate in persons in $\mathrm{HD}$ may reach $50 \%$ at 7 months. The main cause of death is cardiovascular. In conclusion, CKD is a common condition, with problems for making a proper diagnosis and early treatment. There are problems for HD coverage and the mortality rate in persons undergoing $\mathrm{HD}$ is high.
\end{abstract}

Keywords:

Renal insufficiency, chronic; Epidemiology; Renal dialysis (source: MeSH NLM).

\section{INTRODUCCIÓN}

La enfermedad renal crónica (ERC) es un problema de salud pública que está asociada a alta mortalidad cardiovascular (CV) y a altos gastos en los sistemas de salud $[1,2]$. Se estima que a nivel mundial se destinan cerca de 1 triillón de dólares en los cuidados de pacientes con ERC que requieren algún tipo de terapia de reemplazo renal (TRR) [3]. Estos gastos representan un reto en los países de bajos recursos donde los sistemas de salud no se dan abasto para la atención de toda la población que requiere algún tipo de TRR como la hemodiálisis (HD) [4].

En Perú, se estima que más del $50 \%$ de la población que potencialmente requiere algún tipo de TRR no la está recibiendo [5]. Existen regiones del país donde el Ministerio de Salud (MINSA) no cuenta con centros de diálisis en sus hospitales para el manejo de éstos pacientes [5], ni con nefrólogos para su atención [6]. Aunque es un problema que va más allá de recursos económicos, algunas estimaciones sugieren que para dar cobertura similar al promedio latinoamericano a los pacientes que requieren algún tipo de TRR, el presupuesto de salud debería duplicarse [7].

En vista de que a nivel mundial se espera que la población que requiera HD se duplique en los próximos 10 años [8], la International Society of Nephrology (ISN) ha sugerido que la ERC sea incluida dentro de los programas nacionales de enfermedades crónicas no transmisibles [9]. Como parte de estos esfuerzos, el paso inicial debería ser documentar la carga de la ERC en los países, así como delinear las características de los pacientes con ERC [9].

Como parte de ésta iniciativa, en los últimos años se han publicado artículos que exploran la problemática de la ERC en el Perú, siendo necesario sistematizar esta información para poner al alcance de las autoridades del sector salud y a la comunidad médica nacional las cifras que permitan identificar puntos perfectibles en el manejo de ésta enfermedad, con el fin de mejorar la atención de nuestros pacientes.
Por ello, el objetivo del presente artículo, es desarrollar una revisión narrativa de la producción científica que se ha publicado en la literatura médica sobre ERC en población peruana con dirección y filiación Perú hasta abril del 2016. Para la misma, se desarrolló una búsqueda en las siguientes bases de datos: Web of Science Core Collection, Biological Abstracts, BIOSIS Previews, $\mathrm{CABI}$ (CAB Abstracts y Global Health) Data Citation Index (Science (DCI-S) y Social Sciences \& Humanities (DCI-SSH), MEDLINE, LILACS, SciELO, SCOPUS y Google Scholar. Los resultados se presentan en seis áreas temáticas pre establecidas: prevalencia de ERC, etiología de la ERC, diagnóstico y manejo de la ERC, cobertura de la atención de la ERC, costos de la atención en ERC y mortalidad de los pacientes con ERC.

Se encontraron 39 artículos en las bases de datos consultadas. Ocho artículos fueron excluidos ya que no se encontraron textos completos para su lectura y análisis. Luego de la lectura de los mismos, 16 artículos se incluyeron en esta revisión al estar referidos a las áreas temáticas pre establecidas. Las principales características de los mismos se presentan en la Tabla 1.

\section{PREVALENCIA DE LA ENFERMEDAD RENAL CRÓNICA}

Se encontraron dos artículos que hacían referencia a esta área temática.

Cieza et al. (1992) realizó una encuesta entre 15 y el 30 de enero de 1990 en la ciudad de Lima a todos los pacientes que acudieron en dicho periodo a cualquier de los siguientes servicios: unidades de hemodiálisis, centros de emergencia, salas de hospitalización y consultorios externos nefrológicos en todos los hospitales del seguro social (EsSalud), MINSA, Fuerzas Armadas y Policiales; así como en 4 clínicas privadas que mantenían centros de diálisis y en todos los centros de diálisis ambulatorios de la ciudad. Se realizó un cálculo de la prevalencia de pacientes "urémicos" defendiéndolos como los pacientes que tenían una depuración de creatinina menor a $10 \mathrm{ml} / \mathrm{min}$ o creatinina mayor a $5 \mathrm{mg} / \mathrm{dl}$ 
Tabla 1. Características de los estudios incluidos.

\begin{tabular}{|c|c|c|c|}
\hline Autor, año & $\begin{array}{l}\text { Tipo de artículo/ } \\
\text { Diseño }\end{array}$ & Objetivo & Muestra \\
\hline Cieza, 1990[10] & $\begin{array}{l}\text { Original / } \\
\text { Transversal }\end{array}$ & $\begin{array}{l}\text { Determinar la prevalencia de insuficiencia renal } \\
\text { crónica }\end{array}$ & $\begin{array}{l}\text { Registros de los servicios de salud } \\
\text { de Lima }\end{array}$ \\
\hline Cieza, $1992[21]$ & Original / Cohorte & $\begin{array}{l}\text { Explorar los factores asociados a mortalidad } \\
\text { en pacientes con insuficiencia renal crónica } \\
\text { terminal }\end{array}$ & $\begin{array}{l}619 \text { pacientes incidentes de } \\
\text { hospitales de MINSA, EsSalud y } \\
\text { FFAA y clínicas privadas de Lima }\end{array}$ \\
\hline Cieza, 1996 [12] & Original / Cohorte & $\begin{array}{l}\text { Determinar la calidad de atención de salud en } \\
\text { centros de hemodiálisis de Lima }\end{array}$ & $\begin{array}{l}521 \text { pacientes prevalentes de } \\
\text { centros de hemodiálisis de Lima }\end{array}$ \\
\hline Hurtado, 2007 [7] & Revisión & $\begin{array}{l}\text { Describir la enfermedad renal crónica terminal, } \\
\text { sus factores de riesgo en Perú y mostrar la } \\
\text { costo efectividad de la detección temprana de } \\
\text { la misma en población hipertensa }\end{array}$ & - \\
\hline $\begin{array}{l}\text { Meneses Liendo, } \\
2011[13]\end{array}$ & Original / Cohorte & $\begin{array}{l}\text { Comparar la sobrevida de pacientes en } \\
\text { hemodiálisis antes de } 1982 \text { a 1994, y de } 1995 \\
\text { a } 2007\end{array}$ & $\begin{array}{l}359 \text { pacientes prevalentes en un } \\
\text { centro privado de Lima }\end{array}$ \\
\hline $\begin{array}{l}\text { Loza-Concha, } \\
2011[19]\end{array}$ & $\begin{array}{l}\text { Original / Costo } \\
\text { utilidad }\end{array}$ & $\begin{array}{l}\text { Determinar y comparar las razones de costo } \\
\text { utilidad del trasplante renal con donante } \\
\text { cadavérico frente a la hemodiálisis. }\end{array}$ & $\begin{array}{l}\text { Datos de } 5^{8} \text { pacientes prevalentes } \\
\text { de un hospital de EsSalud de Lima }\end{array}$ \\
\hline Cieza, 2013. [22] & Original / Cohorte & $\begin{array}{l}\text { Describir la supervivencia y calidad de vida } \\
\text { de pacientes con enfermedad renal crónica } \\
\text { terminal en terapias de reemplazo renal }\end{array}$ & $\begin{array}{l}175 \text { pacientes prevalentes en un } \\
\text { hospital del MINSA de Lima }\end{array}$ \\
\hline Ramos, 2014 [16] & $\begin{array}{l}\text { Original / } \\
\text { Transversal }\end{array}$ & $\begin{array}{l}\text { Describir los hallazgos de la vigilancia } \\
\text { epidemiológica de diabetes mellitus }\end{array}$ & $\begin{array}{l}2959 \text { pacientes de } 18 \text { hospitales } \\
\text { de Lima, Piura, Tumbes, Ica y } \\
\text { Junín }\end{array}$ \\
\hline $\begin{array}{l}\text { Herrera-Añazco, } \\
2014 \text { [18] }\end{array}$ & Original / Cohorte & Determinar la tasa de abandono de diálisis & $\begin{array}{l}190 \text { pacientes de un hospital del } \\
\text { MINSA de Lima }\end{array}$ \\
\hline $\begin{array}{l}\text { Herrera-Añazco, } \\
2014 \text { [17] }\end{array}$ & $\begin{array}{l}\text { Original / } \\
\text { Transversal }\end{array}$ & $\begin{array}{l}\text { Determinar las características clínicas de los } \\
\text { pacientes diabéticos que acuden a su primera } \\
\text { consulta nefrológica. }\end{array}$ & $\begin{array}{l}200 \text { pacientes de los consultorios } \\
\text { de nefrología de } 04 \text { hospitales del } \\
\text { MINSA de Lima }\end{array}$ \\
\hline $\begin{array}{l}\text { Concepción-Zavaleta, } \\
2015[24]\end{array}$ & $\begin{array}{l}\text { Original / Casos y } \\
\text { controles }\end{array}$ & $\begin{array}{l}\text { Explorar los factores de riesgo para mortalidad } \\
\text { en pacientes con enfermedad renal crónica en } \\
\text { hemodiálisis }\end{array}$ & $\begin{array}{l}164 \text { pacientes prevalentes de } 2 \\
\text { hospitales del MINSA de Trujillo }\end{array}$ \\
\hline $\begin{array}{l}\text { Herrera-Añazco, } \\
2015[23]\end{array}$ & Original / Cohorte & $\begin{array}{l}\text { Explorar los factores de riesgo para mortalidad } \\
\text { intrahospitalaria en pacientes en hemodiálisis }\end{array}$ & $\begin{array}{l}216 \text { pacientes incidentes en un } \\
\text { hospital del MINSA de Lima }\end{array}$ \\
\hline $\begin{array}{l}\text { Herrera-Añazco, } \\
2015[14]\end{array}$ & Original / Cohorte & $\begin{array}{l}\text { Determinar la mortalidad de pacientes con } \\
\text { enfermedad renal crónica en hemodiálisis }\end{array}$ & $\begin{array}{l}235 \text { pacientes incidentes en un } \\
\text { hospital del MINSA de Lima }\end{array}$ \\
\hline Francis, 2015 [11] & $\begin{array}{l}\text { Original / } \\
\text { Transversal }\end{array}$ & $\begin{array}{l}\text { Determinar la prevalencia de enfermedad } \\
\text { renal crónica en Lima y Tumbes, y sus factores } \\
\text { asociados }\end{array}$ & 404 pobladores de Lima y Tumbes \\
\hline $\begin{array}{l}\text { Herrera-Añazco, } \\
2015[15]\end{array}$ & Revisión & $\begin{array}{l}\text { Describir la producción científica peruana } \\
\text { sobre diabetes mellitus } 2 \text { y nefropatía diabética }\end{array}$ & 22 artículos encontrados \\
\hline $\begin{array}{l}\text { Herrera-Añazco, } \\
2015[5]\end{array}$ & Carta al editor & $\begin{array}{l}\text { Describir los problemas de la cobertura de } \\
\text { terapias de reemplazo renal en el Ministerio de } \\
\text { Salud del Perú }\end{array}$ & - \\
\hline
\end{tabular}


o urea mayor a $10 \mathrm{mg} / \mathrm{dl}$ o pacientes en diálisis o con síntomas de uremia. Se calculó una tasa total de pacientes urémicos de 122 pacientes por millón de habitantes, y considerando solo los pacientes que provenían de Lima, una tasa de go pacientes por millón de habitantes, para una población estimada en Lima en ese entonces de 6423000 [10].

Francis et al. (2015), en un estudio poblacional de 404 habitantes de Lima y Tumbes con una edad promedio de 54,9 años siendo la mitad varones, encuentra una prevalencia de ERC de 16,8\% ( $95 \%$ IC 13,5-20,9\%) usando tasa de filtración glomerular (TFG) calculada y albuminuria. La prevalencia fue mayor en Lima que en Tumbes $(20,7 \%$ vs $12,9 \%)$ y estuvo asociada a mayor edad, sexo femenino, diabetes, hipertensión, residir en Lima y mayor tercil de riqueza [11].

\section{ETIOLOGÍA DE LA ENFERMEDAD RENAL CRÓNICA}

No se encontraron artículos que específicamente se refieran a ésta área temática. Sin embargo, en los reportes sobre mortalidad de tres poblaciones en diálisis, se muestran datos de la etiología de la ERC.

Cieza et al. (1996) en un análisis de la calidad de la atención de siete centros de diálisis en Lima describen la etiología de la ERC entre sus pacientes. La principal causa de ERC fue la glomerulonefritis crónica (GNC) con $38 \%$, seguida de la diabetes mellitus (DM) en un $13 \%$ de los casos [12].

Meneses-Liendo et al. (2011) en su análisis de mortalidad de una población prevalente en HD en un centro privado en Lima adscrito a EsSalud, refiere que la principal causa de ERC entre sus pacientes es la GNC primarias con $32 \%$, seguido de la DM con $22 \%$ y la uropatía obstructiva (UPO) y nefritis túbulo intersticial con $13 \%$ [13].

Herrera-Añazco et al. (2015), en su reporte de mortalidad de la población incidente en HD del Hospital Nacional 2 de Mayo de Lima $\left(\mathrm{HN}_{2} \mathrm{M}\right)$ refiere que la principal causa de la ERC entre sus pacientes es la DM con un $44 \%$, seguida de la GNC con $23 \%$ y la UPO con 15\% [14].

\section{DIAGNÓSTICO Y MANEJO DE LOS PACIENTES CON ENFERMEDAD RENAL CRÓNICA}

Los artículos que han referidos aspectos relacionados a esta área temática reflejan que el diagnóstico y manejo de los pacientes con ERC es deficiente y que el despistaje de la misma en poblaciones de riesgo puede ser costo efectiva en pacientes peruanos.

En una revisión no sistemática de la producción científica que sobre DM y nefropatía diabética se ha publicado con población peruana, Herrera-Añazco et al. (2015) describe artículos que dan cuenta de un pobre despistaje de la ERC en pacientes diabéticos [15]. Así, Ramos et al. (2014) en un estudio hecho en 18 hospitales notificantes a nivel nacional, encuentra que solo el $8,9 \%$ de los pacientes diabéticos tenían un examen de albuminuria entre sus evaluaciones [16].

Herrera-Añazco et al. (2014) en un estudio de pacientes diabéticos atendidos en su primera consulta nefrológica en hospitales públicos de Lima, encuentra que el $81,5 \%$ de los pacientes evaluados no refería haber tenido una evaluación previa de la función renal y del 18,5\% que la tuvo, el 40,5\% la tuvo hacía más de un año [17].

Hurtado (2006) desarrolló una evaluación de costo efectividad del despistaje de ERC en pacientes hipertensos. El estudio mostró que en sujetos mayores de 50 años usando tira reactiva para la evaluación de albuminuria, seguida del uso de enalapril en pacientes con proteinuria y evitando la necesidad de TRR, se ahorra $\$ 188518645$ dólares americanos en cinco años y $\$ 19,70$ dólares americanos por paciente por año [7].

Respecto al manejo de los pacientes con ERC, Herrera-Añazco et al. (2014) también reportan que, entre los pacientes diabéticos de su estudio, el 39,5\% de los mismos tenía una $\mathrm{HbA}_{1} \mathrm{C}>$ a $7 \%$. Cerca de la mitad de los pacientes no seguía las indicaciones dietéticas ni tomaba sus medicamentos regularmente. Así mismo, a pesar de que el $70 \%$ de los pacientes era hipertenso, cerca de un $20 \%$ no recibía un inhibidor del sistema renina angiotensina [17].

Herrera-Añazco et al. (2015) describen que entre los pacientes incidentes al programa de $\mathrm{HD}$ crónica del $\mathrm{HN}_{2} \mathrm{M}$, cerca de la mitad de ellos se enteraban de su diagnóstico en el momento mismo de su ingreso a diálisis. De igual forma, sólo el $24 \%$ de los pacientes había recibido evaluación por un nefrólogo en al menos dos oportunidades en el último año [14].

\section{COBERTURA DE LA ATENCIÓN DE LA ENFERMEDAD RENAL CRÓNICA}

Se tiene alguna información de la cobertura de HD en el MINSA, más no de la cobertura de los pacientes de EsSalud y de la cobertura de la atención en los primeros estadios de la ERC.

Herrera-Añazco et al. (2015) menciona que para el 2014, el Fondo Intangible de Solidaridad en Salud (FISSAL), que presta atención a los pacientes del MINSA, tenía 1983 pacientes en cobertura de diálisis a nivel nacional. Estos pacientes junto a los 9814 que reportaba EsSalud en el 2010, representaban en el mejor de los casos, tan solo el $50 \%$ de los pacientes que potencialmente requerían algún tipo de TRR en Perú [5]. Asimismo, se recalcaba que había regiones del país que en ese año no tenía ni hospitales con unidad de diálisis disponibles, ni centros privados contratados por FISSAL para dar atención a los pacientes con ERC que necesitaban HD [5]. 
A pesar de ello, la atención del FISSAL ha mejorado parcialmente la cobertura de la atención en el MINSA, ya que antes de la implementación de ésta institución, el HN2M reportaba que cerca del $50 \%$ de los pacientes con ERC que iniciaban $\mathrm{HD}$ en dicho hospital se retiraban de la terapia en los siguientes seis meses. Siendo la primera causa de ello, la falta de cupos disponibles para dar atención a todos los pacientes que requerían $\mathrm{HD}$ [18].

\section{COSTOS DE LA ATENCIÓN DE LA ENFERMEDAD RENAL CRÓNICA}

Los estudios publicados son referidos a los costos de las TRR y no de los costos de la atención de la ERC en los primeros estadios de la misma.

Loza-Concha et al. (2011) realizó una evaluación de las razones de costo utilidad (CU) del trasplante renal con donante cadavérico (TRDC) vs la HD en Essalud. Los autores encuentran que las razones de CU para los TRDC y los HD fueron de $\$ 11984$ dólares americanos y \$ 9243 dólares americanos, y la razón de CU incremental fue de $\$ 40669$ dólares americanos [19].

Hurtado A. (2006) calcula los costos de la HD vs la diálisis peritoneal (DP) en Perú, la cual resulta en $\$ 594,00$ dólares americanos vs. $\$ 727,00$ dólares americanos, respectivamente [7]. Desafortunadamente, el autor no señala la metodología utilizada para hacer éste cálculo y a pesar de que éste texto no ha sido publicado en una revista científica, el mismo se encuentra disponible en las bases de datos consultadas y ha sido incluido en una revisión de costos de DP y HD a nivel mundial, motivo por lo cual decidimos incluirlo [20].

\section{MORTALIDAD DE LOS PACIENTES CON ENFERMEDAD RENAL CRÓNICA}

Los estudios son referidos a los pacientes en TRR y no a la mortalidad de la ERC en todos los estadios.

Cieza et al. (1992) evalúa la mortalidad de pacientes en HD en hospitales tanto del MINSA, EsSalud, Fuerzas Armadas y Clínicas privadas; desde enero de 1990 hasta enero de 1991. Al final del seguimiento, permanecían vivos el $82 \%$ de los pacientes de clínicas privadas, el $55 \%$ de los pacientes de EsSalud, el $48 \%$ de los pacientes en centros de las Fuerzas Armadas y solo el $4 \%$ de los hospitales de hospitales del MINSA. Sin embargo, este análisis también reconoce que hubo un gran número de pacientes que se retiraron en todas las instituciones, en especial en el MINSA, donde se retiraron el 96\% [21].

De igual forma, Cieza et al. (1996) realiza un análisis de los fallecidos en siete centros de diálisis privados de Lima en 1993 expresado en pacientes-año en riesgo (PAR), y encuentra una tasa de mortalidad de 1,15 por cada 10 PAR [12].

Meneses Liendo et al. (2011) en un análisis de pacientes pertenecientes a un centro de diálisis privados afiliados a
EsSalud, evalúa la mortalidad de sus pacientes dividiéndolos en dos grupos. El grupo 1 estaba formado por los pacientes que ingresaron HD entre el año 1982 hasta 1994 y el grupo 2 formado por los pacientes que ingresaron a HD entre los años $1995 \mathrm{al} 2007$. La supervivencia aproximada al primer y quinto año fue $87 \%$ y $54 \%$ para el grupo $1 ;$ y $86 \%$ y $62 \%$ para el grupo 2 , sin diferencias significativas entre ambos [13]

Cieza et al. (2013) en un análisis de sobrevida de los pacientes en TRR en el Hospital Nacional Cayetano Heredia $(\mathrm{HNCH})$ entre los años 2008 y 2012 reportó que la supervivencia general de los pacientes fue de $95 \%$ al primer año; $92 \%$ al segundo año; $y$ $89 \%$ al tercer año. Específicamente entre los pacientes con $\mathrm{HD}$, la sobrevida en HD fue: $98 \%$ a los seis meses; $95 \%$ al primer año; $94 \%$ al año y medio; $91 \%$ al segundo año; y $88 \%$ al tercer año. $Y$ entre los pacientes en trasplante renal, la sobrevida fue de: $97 \%$ a los seis meses permaneciendo así al primer año y segundo año al no registrarse otra muerte [22].

Herrera-Añazco et al. (2015) realiza un análisis de la mortalidad de los pacientes incidentes en HD durante su primera hospitalización y a mediano plazo en el HN2M. En el primer caso, la tasa de mortalidad fue de 9,3 muertes/100 personas-semanas (IC 95\%: 7,0 a 12,3) [23] y en el seguimiento a mediano plazo, la mortalidad fue de $37,7 \%(95 \%$ IC; 29,3 a 48,5$)$, al tercer mes y de $49,5 \%(95 \%$ IC 38,8 a 61,4$)$ al séptimo mes [14].

Respecto a la causa de mortalidad, Concepción-Zavaleta et al. (2015) en su estudio entre pacientes en HD del MINSA en Trujillo encuentra que la principal causa directa de mortalidad fue la cardiovascular (46\%), seguida de la causa infecciosa y metabólica con $17 \%$ cada una, y neoplásica en el 9,8\% de los casos [24].

\section{DISCUSIÓN}

Los principales resultados de la presente revisión muestran que la enfermedad renal crónica es una patología frecuente en el Perú, con serios problemas de diagnóstico precoz y manejo oportuno en las primeras etapas de la enfermedad. Asimismo, hay problemas de cobertura en la atención de la hemodiálisis a nivel nacional y alta mortalidad en hemodiálisis, siendo la primera causa de ella de origen cardiovascular.

\section{La enfermedad renal crónica como problema de salud pública en el Perú}

Según el 2013 Global Burden of Disease study, la enfermedad renal crónica como causa de muerte en la población general subió del puesto 36 al puesto 19 en dos décadas [25]. Aunque no hay estudios poblacionales al respecto en nuestro país, una reciente carta al editor usando la codificación $\mathrm{CIE}-10$ del MINSA revela que la insuficiencia renal representa el $3,5 \%$ de las muertes a nivel nacional [26]. Este hecho junto a la evidencia de que la prevalencia de ERC en algunas regiones del país está por encima de la media internacional $[11,27]$, y que hay problemas de cobertura de la HD en el Perú [5], muestran la importancia de considerar a la ERC como un problema de salud pública en nuestro país. 
En Perú, se ha planteado estrategias de detección temprana y manejo de estadios iniciales de la ERC centrándose en las poblaciones de riesgo [28]. Así mismo, se ha sugerido que las medidas de detección temprana en población de riesgo son costo efectivas en nuestro país [7]. En ese sentido, resulta necesario que el MINSA diseñe una estrategia integral de alcance nacional para el abordaje de ésta enfermedad [29].

En vista que los dos principales factores de riesgo de la ERC, como son la DM y la hipertensión arterial (HTA) [30], son patologías de prevalencia creciente en nuestro país [31], un manejo multidisciplinario como ha sido sugerido por la Kidney Disease Improving Global Outcomes (KDIGO) debería incluirse en ésta estrategia integral [29,32]. De igual forma, no se debe dejar de lado el aumento de la frecuencia de diagnóstico de las glomerulonefritis en Perú [33], que ha sido reportada como la principal causa de la ERC en HD en algunos estudios en nuestro país $[12,13]$.

\section{Capacitación de médicos peruanos no nefrólogos}

El abordaje integral debe ir de la mano de una capacitación del personal de salud que evaluará a los pacientes en los primeros estadios de la enfermedad [29]. Se espera que la atención del nefrólogo solo sea para casos específicos, sobre todo en la atención de pacientes en estadios avanzados de la ERC $[29,32]$. Esta recomendación cobra especial relevancia cuando se ha sugerido una disminución del interés de los médicos peruanos en seguir la carrera de nefrología a pesar del aumento del número de vacantes para la especialidad en los últimos años [34].

El haber encontrado entre nuestros resultados falta de despistaje de la ERC en pacientes diabéticos [16], pobre control de factores de progresión de la ERC [17], así como falta de referencia oportuna de pacientes con ERC al nefrólogo [14], sugiere deficiencias en la capacitación entre médicos peruanos de temas nefrológicos. No obstante, es un problema que ha sido reportado entre médicos no nefrólogos en otras partes del mundo [35] y que puede contribuir a la falta de autoconocimiento de la ERC en los propios pacientes, que está por encima de los niveles de falta de conocimiento de la DM o HTA en países de bajos recursos [36].

\section{Terapias de reemplazo renal}

Un adecuado manejo de los estadios iniciales de la ERC disminuirá la probabilidad de la progresión a estadios en que los pacientes requerirán alguna forma de TRR como la HD [32]. Lo que es relevante debido a los problemas de cobertura de la misma a nivel nacional y los costos que implican para un sistema de salud deficiente como el nuestro [4,5].

A pesar de que en muchos países del tercer mundo la DP ha sido sugerida como la TRR más económica y ha sido impulsada en Perú $[20,37,38]$. En el análisis económico, esta ha resultado más cara comparada con la HD $[7,20]$. Si bien es cierto los costos han variado desde la fecha en que Hurtado hizo el cálculo en nuestro país [7], no han variado las condiciones que se han señalado como las causas para que en algunos países la DP sea más cara que la HD. Así, se ha sugerido que la necesidad de exportar los insumos para la misma y el menor costo en salarios para el personal de salud que trabaja en HD en comparación con otros países que la impulsan, son las razones por las que ésta es más cara en algunos países como el Perú $[7,20]$. De igual forma, aunque el trasplante renal en general es la medida más costo efectiva a nivel mundial y se impulsa su desarrollo [37], en nuestro país, al menos entre pacientes de EsSalud, el TRDC es menos costo efectiva que la HD [19]. Sin embargo, aún queda pendiente el análisis del trasplante con donante vivo relacionado, que entre los donantes para el trasplante renal, es el que se ha asociado a mejores resultados en términos de sobrevida general y renal así como el ser más costo efectivo en comparación al TRDC [37].

Solo un estudio evalúa las causas de mortalidad de la población en $H D$, y al igual que la literatura internacional, las causas CV son las más frecuentes $[24,40,41]$. A pesar de ser realizado en población del MINSA, no hay motivos para pensar que la población de EsSalud sea diferente, debido a que, entre sus pacientes, la carga de enfermedad CV puede llegar hasta el $82 \%$ [42].

Las discrepancias entre las cifras de mortalidad entre los estudios de pacientes en HD se pueden explicar por la selección de población estudiada [12-14,21,22]. Sin embargo, llama la atención que en algunos reportes, la mortalidad sea alarmantemente alta [14]. Las razones de esta alta mortalidad están en relación con un deficiente sistema de atención antes de la llegada a $\mathrm{HD}$, referido a una pobre detección precoz de la ERC y referencia tardía al nefrólogo para su evaluación [14,23]. Estos hallazgos vuelven a reforzar la idea de una estrategia nacional integral de la atención de éstos pacientes.

\section{LIMITACIONES}

La presente revisión narrativa tiene algunas limitaciones. En primer lugar, solo se han incluido datos de estudios publicados en revistas científicas, pudiendo existir datos de tesis, reportes técnicos, etc, que podrían dar una visión más completa de la ERC en el país.

En segundo lugar, los estudios incluidos tienen serias limitaciones metodológicas que incluyen los siguientes aspectos: muchos de los estudios son de un solo centro, tienen poca muestra y ésta generalmente es por conveniencia. Los resultados en muchos casos no son generalizables a la población de EsSalud, hospitales de las Fuerzas Armadas u pacientes de seguros privados, ya que la mayoría de estudios son hechos en población del MINSA, lo que llama la atención, debido a que la mayor cantidad de pacientes en HD están en EsSalud. La mayoría de los estudios son hechos en población limeña lo que no permite extrapolar los resultados a otras regiones del país, no obstante, la gran cantidad de facultades de medicina en provincia con potenciales investigadores en ésta área.

En tercer lugar, hay otros estudios hechos en pacientes con ERC, sin embargo, se dejaron de lado al no estar referidos a las áreas 
temáticas de ésta revisión. En cuarto lugar, los estudios de costos deberían actualizarse al sistema de pagos actual en las distintas instituciones en las que se presta terapias de reemplazo renal.

A pesar de ello, es la primera revisión de este tipo sobre la ERC en el Perú y nos permite tener una visión integral sobre puntos importantes a evaluar en futuras investigaciones con mejor diseño metodológico.

En conclusión, la ERC es una patología frecuente, con problemas en al diagnóstico temprano y manejo de los primeros estadios de la enfermedad. La cobertura de HD es deficiente a nivel nacional y la mortalidad de los pacientes en HD puede ser muy alta.

\section{Fuente de financiamiento:}

Los autores declaran no haber recibido ninguna financiación para la realización de este trabajo.

\section{Declaración de conflicto de intereses:}

Los autores declaran no tener conflicto de intereses con la publicación de este artículo.

\section{REFERENCIAS BIBLIOGRÁFICAS}

1. James MT, Hemmelgarn BR, Tonelli M. Early recognition and prevention of chronic kidney disease. Lancet. 2010;375(9722):1296309.

2. Sarnak MJ, Levey AS, Schoolwerth AC, Coresh J, Culleton B, Hamm LL, et al. Kidney disease as a risk factor for development of cardiovascular disease. A statement from the American Heart Association councils on kidney in cardiovascular disease, high blood pressure research, clinical cardiology, and epidemiology and prevention. Circulation. 2003;108(17):2154-69.

3. Stenvinkel P. Chronic kidney disease: a public health priority and harbinger of premature cardiovascular disease. J Intern Med. 2010;268(5):456-67.

4. Mushi L, Marschall P, Fleßa S. The cost of dialysis in low and middle income countries: a systematic review. BMC Health Serv Res. 2015;15:506.

5. Herrera-Añazco P, Benites-Zapata VA, León-Yurivilca I, HuarcayaCotaquispe R, Silveira-Chau M. Chronic kidney disease in Peru: a challenge for a country with an emerging economy. J Bras Nefrol. 2015;37(4):507-8.

6. Zevallos $L$, Pastor R, Moscoso B. Oferta y demanda de médicos especialistas en los establecimientos de salud del Ministerio de Salud: brechas a nivel nacional, por regiones y tipo de especialidad. Rev Peru Med Exp Salud Publica. 2011;28(2):177-85

7. Hurtado A. End stage renal failure and risk factors in Peru [documento en Internet]. Lima: Ministerio de Salud; 2006 [citado el 16 de mayo de 2016]. Disponible en: ftp://ftp2.minsa.gob.pe/ donwload/esn/dnt/srenal/InsRenalPeru.pdf

8. Liyanage $T$, Ninomiya $T$, Jha $V$, Neal B, Patrice HM, Okpechi I, et al. Worldwide access to treatment for end-stage kidney disease: a systematic review. Lancet. 2015;385(9981):1975-82.

9. Tonelli M, Agarwal S, Cass A, Garcia Garcia G, Jha V, Naicker S, et al. How to advocate for the inclusion of chronic kidney disease in a national non-communicable chronic disease program. Kidney Int. 2014;85(6):1269-74
10. Francis ER, Kuo CC, Bernabe-OrtizA, Nessel L, Gilman RH, Checkley $W$, et al. Burden of chronic kidney disease in resource-limited settings from Peru: a population-based study. BMC Nephrol. 2015;16:114.

11. Cieza J, Huamán C, Alvarez C, Gómez J, Castillo W. Prevalencia de insuficiencia renal crónica en la ciudad de Lima-Perú, enero 1990. Rev Peru Epidemiol. 1992;5(1):22-7.

12. Herrera-Añazco $P$, Benites-Zapata $V$, Hernandez AV, MezonesHolguin $E$, Silveira-Chau M. Mortality in patients with chronic kidney disease undergoing hemodialysis in a public hospital of Peru. J Bras Nefrol. 2015;37(2):192-7.

13. Meneses Liendo V, Rabanal Leon C, Huapaya Carrera J, Cieza Zevallos J. Sobrevida en hemodiálisis según el periodo de ingreso de pacientes entre 1982 y 2007 en Lima, Perú. Rev Med Hered. 2011;22(4):157-61.

14. Cieza J, Loza C. Evaluación de la calidad de los servicios de hemodiálisis según edad y etiología diabética. Nefrologia. 1996;16(5):439-45.

15. Herrera Añazco P, Hernandez AV, Mezones-Holguin E. Diabetes mellitus y nefropatía diabética en el Perú. Rev Nefrol Dial Transpl. 2015;35(4):229-37.

16. Ramos W, López $T$, Revilla L, More L, Huamaní M, Pozo M. Resultados de la vigilancia epidemiológica de diabetes mellitus en hospitales notificantes del Perú, 2012. Rev Peru Med Exp Salud Publica. 2014;31(1):9-15

17. Herrera-Añazco $P$, Bonilla Vargas $L$, Palacios Guillén $M$, Valencia Rodríguez J, Sánchez Riva F, Salomé Luna J, et al. Características clínicas de los pacientes diabéticos que acuden por primera vez a una consulta nefrológica en hospitales públicos de Lima. An Fac Med (Lima). 2014;75(1):25-9.

18. Herrera-Añazco $P$, Palacios Guillén $M$, Hernández AV. Alta tasa de interrupción de hemodiálisis en pacientes del hospital nacional 2 de mayo de Perú. Rev Nefrol Dial Transpl. 2014;34(2):94-8.

19. Loza-Concha R, Quispe AM. Costo-utilidad del trasplante renal frente a la hemodiálisis en el tratamiento de la insuficiencia renal crónica terminal en un hospital peruano. Rev Peru Med Exp Salud Publica. 2011;28(3):432-9.

20. Karopadi AN, Mason G, Rettore E, Ronco C. Cost of peritoneal dialysis and haemodialysis across the world. Nephrol Dial Transplant. 2013;28(10):2553-69.

21. Cieza J, Bonilla J, Huaman C, Renold T, Castillo W. Factores asociados al pronóstico del paciente con insuficiencia renal crónica terminal en la ciudad de lima. Rev Peru Epidemiol. 1992;5(2):5-9.

22. Cieza J, Hurtado B, Montes Z, Soriano V, Rabanal C. Supervivencia en terapias de reemplazo renal dentro de un concepto integral de oferta de servicios públicos en el Perú, periodo 2008 y 2012. Acta Med Peru. 2013;30(4):80-5

23. Herrera-Añazco $P$, Benítes-Zapata $V$, Hernandez AV. Factores asociados a mortalidad intrahospitalaria de una población en hemodiálisis en el Perú. Rev Peru Med Exp Salud Publica. 2015;32(3):479-84.

24. Concepción-Zavaleta M, Cortegana Aranda J. Ocampo Rugel N, Gutiérrez Portilla W. Factores de riesgo asociados a mortalidad en pacientes con enfermedad renal crónica terminal. Rev Soc Peru Med Interna. 2015;28(2):72-8.

25. GBD 2013 Mortality and Causes of Death Collaborators. Global regional, and national age-sex specific all-cause and cause-specific mortality for 240 causes of death, 1990-2013: a systematic analysis for the Global Burden of Disease Study 2013. Lancet. 2015;385(9963):117-71. 
26. Herrera-Añazco P, Willer H, Taype-Rondan A. Exposición a arsénico como factor asociado a la alta mortalidad atribuida a insuficiencia renal en Puno [carta]. Rev Peru Med Exp Salud Publica. 2016;33(2):381-2.

27. McCullough K, Sharma P, Ali T, Khan I, Smith WC, MacLeod A, et al. Measuring the population burden of chronic kidney disease: a systematic literature review of the estimated prevalence of impaired kidney function. Nephrol Dial Transplant. 2012;27(5):1812-21.

28. Francis ER, Allen AK, Herrera-Añazco P, Kuo CC, Cardenas MK, et al. Establishing a higher priority for chronic kidney disease in Peru. Lancet Glob Health. 2016;4(1):e17-8.

29. Wouters OJ, O'Donoghue DJ, Ritchie J, Kanavos PG, Narva AS. Early chronic kidney disease: diagnosis, management and models of care. Nat Rev Nephrol. 2015;11(8):491-502.

30. Chen RA, Scott S, Mattern WD, Mohini R, Nissenson AR. The case for disease management in chronic kidney disease. Dis Manag. 2006;9(2):86-92.

31. Villena J. Diabetes mellitus in Peru. Ann Glob Health. 2015;81(6):765-75

32. Kidney Disease - Improving Global Outcomes (KDIGO). KDIGO 2012 clinical practice guideline for the evaluation and management of chronic kidney disease. Kidney Int Supplement. 2013;3(1):1-150.

33. Castillo Zegarra ME, Matsuoka Sato J, Asato Higa C, Sumire Humeres J, Rojas Rivera J, Hurtado Aréstegui A. Glomerunefritis primarias: frecuencia de presentación en el período 1996 y 2005 , en Lima, Perú. Rev Soc Per Med Inter. 2005;18(1):15-21.

34. Herrera-Añazco P, Mezones-Holguin E, HernandezAV. Nephrology: is a medical specialty unattractive to Peruvian doctors? J Bras Nefrol. 2014;36(1):102-3.
35. Israni RK, Shea JA, Joffe MM, Feldman HI. Physician characteristics and knowledge of CKD management. Am J Kidney Dis. 2009;54(2):238-47.

36. Ene-lordache B, Perico N, Bikbov B, Carminati S, Remuzzi A, Perna $A$, et al. Chronic kidney disease and cardiovascular risk in six regions of the world (ISN-KDDC): a cross-sectional study. Lancet Glob Health. 2016;4(5):e307-19.

37. Klarenbach SW, Tonelli M, Chui B, Manns BJ. Economic evaluation of dialysis therapies. Nat Rev Nephrol. 2014;10(11):644-52.

38. Atapour A, Eshaghian A, Taheri D, Dolatkhah S. Hemodialysis versus peritoneal dialysis, which is cost-effective? Saudi J Kidney Dis Transpl. 2015;26(5):962-5.

39. Vélez-Segovia E, Salazar-Huayna L, Alva-Bravo E. Peritoneal dialysis in Peru. Indian J Nephrol. 2014;24(1):67-8.

40. Collins AJ, Foley RN, Chavers B, Gilbertson D, Herzog C, Johansen K, et al. United States Renal Data System 2011 Annual Data Report: Atlas of chronic kidney disease \& end-stage renal disease in the United States. Am J Kidney Dis. 2012;59(1 Suppl 1):A7, e1420.

41. Cusumano AM, Gonzalez Bedat MC, García-García G, Maury Fernandez S, Lugon JR Poblete Badal $\mathrm{H}$, et al. Latin American dialysis and renal transplant registry: 2008 report (data 2006). Clin Nephrol. 2010;74 Suppl 1:S3-8.

42. Bravo-Jaimes K, Whittembury A, Santivañez V. High prevalence of cardiovascular disease in end-stage kidney disease patients ongoing hemodialysis in Peru: Why should we care about it? Int J Nephrol. 2015;2015:568702.

\title{
Ahora puede enviar sus artículos para Acta Médica Peruana en nuestro Open Journal System:
}

\author{
www.amp.cmp.org.pe
}

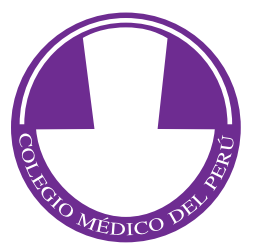

